\title{
Exploring Proper Names Online and Its Application in English Teaching in University
}

\author{
Liqun Liang, Qian Yin and Chunrang Shi \\ ${ }^{1}$ School of English Studies, Xi 'an International Studies University 710128 Xi'an, China \\ Corresponding author: Chunrang Shi (scr200309@aliyun.com)
}

Manuscript submitted 3 May 2021; Accepted 19 May 2021; Published 19 May 2021

Academic Editor: Jinchao Chen

Abstract: Explaining the proper names on the website to students is an essential part of English teaching in university, but it is often overlooked by teachers and students. In the university, students use improper methods of exploring proper names on websites, which include exploring proper names on just a few websites, exploring proper names on inappropriate websites, and improper methods of recognizing the meanings of proper names. Students' improper methods of recognizing the meanings of proper names consist of interpreting out of context, and interpreting by literal meaning. The teachers can guide students to explore proper names online appropriately by using the effective methods, including introducing of relevant websites to lead students to choose the appropriate websites for exploring, comparing the different explanations of proper names from different websites, and questioning students to help them to cognize the accurate understanding of proper names.

Index Terms: Proper Names, English Teaching in University, Exploring Words on the Website.

\section{Introduction}

Vocabulary is the basis of language. It is argent for the teachers to improve the effect of vocabulary teaching. [7] The teaching of proper names is a significant part in vocabulary teaching and the students will often encounter the proper names in English learning in university. These proper names, full of unique cultural or technological information, include all kinds of names of places, people, companies, works, organizations and so on. So it is usually difficult for the students to understand them. In order to understand these proper names quickly, the students can explore the proper names online to get the corresponding explanations, which is the most convenient and efficient way. However, when teaching English in university, the teachers find that the students are unable to find the correct explanations accurately and efficiently by exploring on different websites so that they can not get a correct understanding of these proper names. Therefore, teachers should take the responsibility to teach students how to explore for these proper names on relevant websites and understand their meanings accurately.

2. Students' improper methods of exploring proper names on websites

2.1 Exploring proper names on just a few websites

There are many websites on the Internet as well as engines that are commonly used. Some students are only familiar with a few websites, so they usually just explore on those 
websites to look up proper names instead of trying more websites. Different websites may provide different interpretations of the same proper names. So the information the students got may be lopsided if they just explore on a few websites.

For example, "linguistics" is a proper name that has a complex meaning. If the students do not explore it on several websites, they may just get a lopsided information. When exploring this proper name in Youdao website, we will get this definition"Linguistics is the scientific study of language and it is the humanistic study of language and literature as well." This definition provided by Youdao website is incomprehensive. In the pried of learning, the students need to know the focus of linguistic study, the scope of linguistics, some famous linguists and so on. That means the students need to explore on more websites to get a full understanding of this proper name rather than just exploring it on Youdao dictionary.

\subsection{Exploring proper names on inappropriate websites}

Students often fail to find useful information about proper names because they fail to explore on appropriate websites. Different websites provide different interpretations of the same proper names. Some inappropriate websites may provide the information that are inappropriate even null. Moreover, some of the information are ambiguous or conflicting with each other.

The authoritative websites like Baidu and government websites can provide more specific and authoritative information. Some websites like Google and Baidu can provide general information of proper names while some professional websites can provide professional and detailed information of proper names. And the official websites can provide authoritative explanations that have practical significance.

For example, "Leech" is a name of a great linguist. But if the students explore it on inappropriate websites, they may get a totally wrong definition. The Collins provides the explanations as the Picture 1. In this picture, we can see that leech is just a kind of animal which has no relationship with the name of person. Obviously, this definition is not the one that we need to know. Because we can not find the appropriate explanation on this website, so we need to explore it on another website.

Picture 1 The Definitions of Leech Provided by Collins

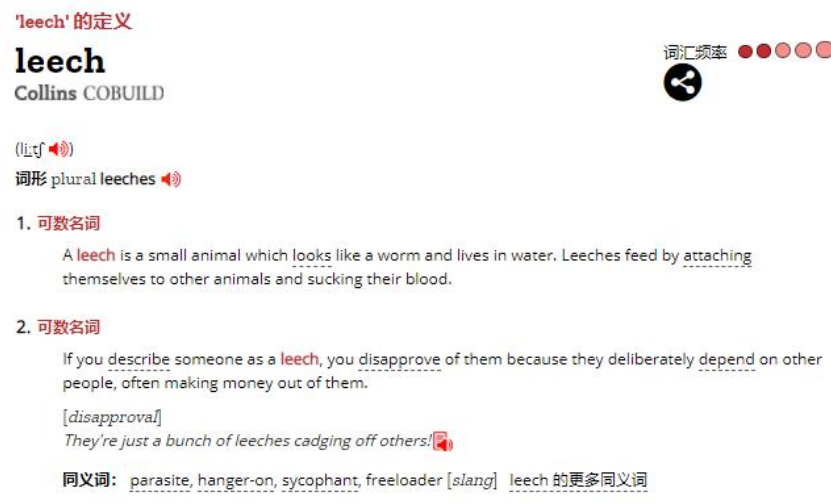

2.3 Frequently exploring the same proper names on websites

Some students don't take the process of exploring information online seriously. After students have found the relevant information of proper names online, they usually just get the literal meaning without the willing to think about their underlying information. In this way, students will not really understand the meanings of the proper names because they do not try to get a deep understanding of the proper names after looking up the relevant information. That means they will look up the same proper names again and again if they come across it again. Then the students will waste more time and energy in exploring the same proper names.

\section{Students' improper methods of recognizing the meanings of proper names}

\subsection{Interpreting out of context}

Some websites may provide an extremely long explanation of proper names. But when some students are reading the information of proper names online, they often only look at the information in the front of the explanation and ignore the rest information. With the idea that they can understand the whole information by just reading a little of them, they will choose the information that they like or understand. Actually, this way does not work. 
For example, The Renaissance is a proper name that refers to a particular period. Wikipedia provides a extremely long explanation about this proper name. The first paragraph of the explanation is as the following: The Renaissance (UK: /rI'neIsəns/ rin-AYsənss, US: /'renəsa:ns/ ([öj]listen) REN-ə-sahnss) was a period in European history marking the transition from the Middle Ages to modernity and covering the 15th and 16 th centuries. It occurred after the Crisis of the Late Middle Ages and was associated with great social change. In addition to the standard periodization, proponents of a "long Renaissance" may put its beginning in the 14th century and its end in the 17 th century. The traditional view focuses more on the early modern aspects of the Renaissance and argues that it was a break from the past, but many historians today focus more on its medieval aspects and argue that it was an extension of the Middle Ages.

Through the above explanations, we can know the time of Renaissance and the research focus. But these two information can not describe a full portrait of Renaissance. If the students just read the first paragraph and ignore the rest paragraph, they will get the superficial meanings rather than more abstract meanings. These more abstract meanings include its spirit core, its cultural effect, its development and so on. Only when we read all the information can we think that we have gotten a full understanding of the Renaissance.

\subsection{Interpreting by literal meaning}

When students interpret information in a literal way without real understanding, they may make a variety of mistakes, of which the most important ones are the following three.

Firstly, some students may only understand the superficial meanings of proper names without understanding its deep meanings. The meanings of some proper names may be so complicate that students are unable to understand it deeply. Because the students are just willing to understand the superficial meanings rather than the deeper meanings, they will only get the superficial information that is disadvantages to their learning. Moreover, with the first sight of the proper names, the students may guess the meaning and consider the guessed meaning correct and appropriate. But, in fact, those guessed meaning are often inappropriate even wrong.
For example, the students will encounter the learning of Chinese zodiacs. Dragon, a kind of Chinese zodiacs, is a proper name as well. At first, Dragon is a kind of imaged animal but it has unique cultural meaning, which is the superficial meaning. It stands for power and status in Chinese culture while it stands for evil in western culture. Dragon is considered as a great creature full of aggressiveness in western culture.[2] If the students just know its superficial meaning and ignore its cultural meaning, they will get an inappropriate understanding of Dragon. This inappropriate understanding is harmful for their cultural learning and social contact.

Secondly, students fail to find the proper explanations of proper names. The British linguist Leech pointed out that words have seven kinds of meanings, including conceptual meaning, connotative meaning, social meaning, affective meaning, reflected meaning, collocative meaning and thematic meaning.[5] So some proper names may also have multiple meanings that may confused the students. The students are required to evaluate and determine which meaning is the one that they really need to know. When the students are trying to understand the proper names, they should focus on all the seven meanings instead of paying attention to only one aspect.

For example, Water-Splashing Festival is an important date in which people splash water with each other. This is just the conceptual meaning. Usually, festivals are not just the days that happen in a fixed date. Just like Chinese festivals, the western festivals also have profound connotations and denotations which are more abstract.[1] When trying to understanding a kind of festival deeply, the students should explore its connotative meaning as well. So the students should also explore the connotative meaning of Water-Splashing Festival. After exploring this proper name deeply, the students will find that this festival is a symbol of luck. People will be lucky because they had washed away the unfortunate things with the splashed water.

Thirdly, students fail to relate the meanings of proper names explored online with the meanings in specific contexts so that they fail to understand the contextual meanings of proper names accurately. Wang Zhanfu had pointed out that the meanings of a word are distinct and clear when the word is put in a particular context. In this 
case, its meanings are not vague and it can be explained just in one way.[12]

For example:Along the way, FedEx pioneered centralization and the "hub and spoke" system, which has since been adopted by almost all major airlines.

The meaning of a sentence is not just a simple combination of its component words.[3] So is the proper name. In this sentence, the word "hub-and-spoke" has a unique contextual meaning. The meaning of this proper name is not the simple combination of the meanings of the three component words, "hub"、 "and"、 "spoke". It refers to the typically US model of passengers being processed through large "hub" airports and then on to secondary flights to "spoke" airports near their final destination.

There is another example: Every white man there had this thought stowed somewhere or other in his mind. I had it, so had the other onlookers, so had the officers on their sweating chargers and the white NCOs marching in the ranks.[11]

In this sentence, NCOs is a proper name that most of the students do not know its meaning. When exploring it on Youdao website, the students will get the following explanation as the picture 2 shows.

\section{Picture 2 The Definitions of NCOs Provided by Youdao} Website

\section{NCOs}

\section{\ا \\ 士官 (non-commissioned officer的复数形式) \\ 网络中心战 (network-centric operation的复数形式) \\ 数控振荡器 (numerically controlled oscillator的复数形式)}

Youdao Website provides three kinds of meanings of NCOs. In order to know its exact meaning in the above sentence, the students need to put the three meanings in the context in sequence and decide which one is fit to the sentence. Then the students can relate its meanings to the meanings in specific contexts so that they could understand the contextual meanings of this proper name accurately.

\section{Guides for students to explore proper names online appropriately}

The ability to explore is the key element of acquiring knowledge and saving time. It focuses on the efficiency of acquiring effective knowledge. And the efficiency of acquiring effective knowledge is a problem that the traditional IQ and EQ can not solve. Therefore, it is also the third ability that human beings need to have in the information age. [13]

That means the ability to explore is extremely important so that the teachers should teach the students how to explore information. In the following part, we will talk about the methods to get effective information by exploring websites.

\subsection{Introduction of relevant websites to lead students to} choose the appropriate websites for exploring

There are always some problems when the students need to choose the appropriate websites for exploring proper names. So teachers should be able to find the problems timely when teaching. They should analyze the reasons why the students choose the improper website and teach them how to choose the appropriate website to look up the information of proper names. Meanwhile, the teacher should explain the reasons to the students patiently. Because only when the students can master correct way to interpret the information on the website can they get the accurate information of the proper names. The teachers should also describe the different features of different websites and teach the students how to find some special and latest information on the websites. In addition, teachers should also guide students to find some latest information that is not available on some websites.

\subsection{Comparing the different explanation of proper names from different websites}

Yuan Hong has pointed out that people who has the higher ability to explore usually explore on many different websites rather than focusing on just one website.[8] Therefore, the students should learn to use more websites for exploring. So teachers should guide students to look up proper names from different websites and then extract those information to compare the different information given by different websites. Then they can determine which one is useful so as to get a fuller and more accurate understanding of this proper name. 
For example, we have explored the word "greenhouse effect" on different websites and get two different definitions.

Definition 1: The greenhouse effect is a process that occurs when gases in Earth's atmosphere trap the Sun's heat. This process makes Earth much warmer than it would be without an atmosphere. The greenhouse effect is one of the things that makes Earth a comfortable place to live.

Definition 2: The greenhouse effect is a natural process that warms the Earth's surface. When the Sun's energy reaches the Earth's atmosphere, some of it is reflected back to space and the rest is absorbed and re-radiated by greenhouse gases. Greenhouse gases include water vapour, carbon dioxide, methane, nitrous oxide, ozone and some artificial chemicals such as chlorofluorocarbons (CFCs). The absorbed energy warms the atmosphere and the surface of the Earth. This process maintains the Earth's temperature at around 33 degrees Celsius warmer than it would otherwise be, allowing life on Earth to exist.

The first definition is concise while the second definition is more concrete. The second definition describes how the Earth's atmosphere is warmed and what are greenhouse gases. And it also provides data to explain why the greenhouse effect makes the Earth a comfortable place to live. So after comparing the two definitions, the students will get a fuller and more accurate understanding of this proper name.

\subsection{Putting questions to help students get more accurate understanding of the proper names online}

Putting questions is an extremely useful English teaching method. It is one of the teaching methods used in the interactive teaching mode and it became popular in France after the 1980s.[10] In the process of teaching, the teachers should use their logical words to lead students into the world of the text. With the guidance of teacher, the students should understand this topic step by step.[6] And putting questions is the most efficient way to guide students. That is to say, the process of question-and-answer is necessary in class. In the process of question-and-answer, the students can get a more accurate understanding of some proper names. By asking kinds of questions, the teachers can lead the students to get a full understanding of all the meanings that the proper names comprise.
For example: In some urban centers, workaholism is so common that people do not consider it unusual: They accept the lifestyle as normal. Government workers in Washington, D.C. for example, often work sixty to seventy hours a week.

Washington, D.C is a proper name in this sentence. Although it is commonly used, many students do not know its accurate meanings.

Then the teachers can put the following questions and ask for answers.

(1)What is the full name of Washington, D.C?

(2)What is the meaning of the component words in its full name?

(3) What is the difference between Washington and Columbia? Why do they appear in the same phrase?

(4)What is the Chinese translation of the capital city of America?

(5)Where is the capital of America precisely? There are fifty states in America. Is the capital city in any one of them?

(6)What's the difference between Washington State and the capital Washington, D.C?

Through asking questions, the teacher can help students to get a full understanding of the capital city of America. More specifically, students will get the knowledge of word formation and cultural features of geographical proper names in English and they will also know some information about American politics, government and geography. In the process of question-and-answer, the students will find the diversity of the information contained in the word "Washington, D.C" and understand why this word is relatively difficult to understand. Meanwhile, the students will find it interesting and worthwhile to do the work of question-and-answer. This is also a process of exploration, which is beneficial for the students to improve their ability of thinking, learning and understanding of vocabulary.

\section{Conclusion}

The teaching of proper names is an essential part of English teaching in university, but it is often overlooked by teachers and students. Actually, it is an efficient and convenient way for students to learn proper names through the way of exploring on websites taught by teachers. Teachers should 
explore more methods in the practice of teaching to help students improve their skills in exploring proper names and their ability to understand the proper names accurately.

\section{Acknowledgement}

This work was supported by $\mathrm{Xi}$ 'an International Studies University (Fund No. 20BY03) , and China National Committee for Terms in Sciences and Technologies (Fund No. YB20200014).

\section{References}

[1]Chen, K. F.(2005). The Cultural Exchange and The Connotation of Western Festivals. Journal of Chengdu University (The Edition of Social Technology) (04):73-75

[2]Ge, C. Y.(2011).The Images of The Western Dragon and The Chinese Dragon and Their Foreign Exchange. Journal of Puyang Technical College(01),1-2.

[3]Hu, Z. L.(2017).Linguistics. Beijing: Peking University Press.

[4]Liu, J. J.(2016).The Case Study of Putting Questions Methods--Take the Advanced Chinese, Offered in a College For The Overseas Students From Duck University. Journal of Yunnan Normal University(The edition of the teachings and researches of Teaching Chinese to Foreigners) (03),45-52.

[5]Leech, G. (1974/1981).Semantics. Harmondsworth: Penguin.

[6]Qi, Q.(2017).Teaching Methods: The Explanation of Concepts. The Study of Education(01),95-105.

[7]Xiao, L.(2021).A discussion of the effective teaching of Vocabulary in University. Overseas English(04),150-151.

[8]Yuan, H. Li, Q.(2015).The Influence of Task and Exploring Ability on The Exploratory Behavior of Users. Information Work in Library (15):94-104

[9]Zhu, Y. F.(2005). The Seven Kinds of meanings and the Teaching of English Vocabulary. Foreign Languages and Their Teaching(09),28-30+35.

[10]Zhou, X. Zhou, Y.(2002). The Research and Analysis of the words of Teachers in University. Foreign Language Teaching and Research(01),59-68.

[11]Zhang, H. X.(2017). Advanced English. Beijing: Foreign Language Teaching and Research Press
[12] Wang, Z.F. (1998).Introduction to Chinese Context. Guangzhou: Nanfang Press.

[13]Wang, H, S. Zhang, Z, C.(2018).A Study of The Ability of the Translators to Explore in The Age of Big Data. Chinese Science \& Technology Translators Journal(04):26-29 